\title{
NEUTRON CAPTURE EXPERIMENTS ON UNSTABLE NUCLEI
}

Annual Technical Report for the Period November 2003-November 2004 Issued: January 2005

Jon M. Schwantes*, Ralf Sudowe, Charles M. Folden III, Heino Nitsche, and Darleane C. Hoffman

Heavy Element Nuclear and Radiochemistry Group Department of Chemistry, University of California, Berkeley and

Lawrence Berkeley National Laboratory

Berkeley, California 94720

*Current affiliation: Isotope \& Nuclear Chemistry Group, Chemistry Division Los Alamos National Laboratory Los Alamos, New Mexico 87545

\author{
PREPARED FOR THE UNITED STATES \\ NATIONAL NUCLEAR SECURITY AGENCY \\ Stewardship Science Academic Alliances Program
}

National Nuclear Security Agency Project NS00075

Award Number DE-FG03-03NA00075 


\section{Project Participants}

Time funded

Dr. Jon M. Schwantes

Postdoctoral Fellow

$100 \%$

Dr. Ralf Sudowe

Term Staff

$17 \%$

Dr. Charles M. Folden III ${ }^{*}$

Postdoctoral Researcher

$100 \%$

*Supported as a graduate student at 4\% time through November 4, 2005.

\section{Principle Investigators}

Time funded

Prof. Darleane C. Hoffman, Professor of the Graduate School, $0 \%$

Department of Chemistry, University of California, Berkeley (Travel expenses to LANL and SSAAP meetings funded).

Prof. Heino Nitsche, Professor of Chemistry, $0 \%$

Department of Chemistry, University of California, Berkeley 


\title{
NEUTRON CAPTURE EXPERIMENTS ON UNSTABLE NUCLEI
}

\author{
Annual Technical Report for the Period November 2003 -November 2004 \\ Issued: January 2005
}

\author{
Jon M. Schwantes, Ralf Sudowe, Charles M. Folden III, Heino Nitsche, and \\ Darleane C. Hoffman
}

\section{EXECUTIVE SUMMARY}

The overall objective of this project is the measurement of neutron capture cross sections of importance to stewardship science and astrophysical modeling of nucleosynthesis, while at the same time helping to train the next generation of scientists with expertise relevant to U.S. national nuclear security missions and to stewardship science. A primary objective of this project is to study neutron capture cross sections for various stable and unstable isotopes that will contribute to the Science Based Stockpile Stewardship (SBSS) program by providing improved data for modeling and interpretation of nuclear device performance. Much of the information obtained will also be important in astrophysical modeling of nucleosynthesis. Measurements of these neutron capture cross sections are being conducted in collaboration with researchers at the Los Alamos Neutron Science Center (LANSCE) facility using the unique Detector for Advanced Neutron Capture Experiments (DANCE). In our early discussions with the DANCE group, decisions were made on the first cross sections to be measured and how our expertise in target preparation, radiochemical separations chemistry, and data analysis could best be applied.

The initial emphasis of the project was on preparing suitable targets of both natural and separated stable europium isotopes in preparation for the ultimate goal of preparing a sufficiently large target of radioactive ${ }^{155} \mathrm{Eu}\left(\mathrm{t}_{1 / 2}=4.7\right.$ years) and other radioactive and stable species for neutron cross-section measurements at DANCE. Our Annual Report, "Neutron Capture Experiments on Unstable Nuclei” by J. M. Schwantes, R. Sudowe, C. M. Folden III, H. Nitsche, and D. C. Hoffman [1], submitted to NNSA in December 2003, gives details about the initial considerations and scope of the project.

During the current reporting period, electroplated targets of natural Eu together with valuable, stable, and isotopically pure ${ }^{151} \mathrm{Eu}$ and ${ }^{153} \mathrm{Eu}$, and isotopically separated ${ }^{154} \mathrm{Sm}$ were measured for the first time at the DANCE facility in early 2004. The Eu targets, suitable blanks, Be backing foils, and standards had been sent to the DANCE group in early fall 2003. Some preliminary data analysis was performed and more sophisticated analysis has begun. We developed plans for 
a suitable computer system for data analysis within our group at Berkeley and had meetings with counterparts at Lawrence Livermore National Laboratory (LLNL) and LANL concerning analysis of these data.

Our major emphasis in 2004 has been to develop the separations and processes ultimately required to prepare radioactive targets of 4.7 -year ${ }^{155} \mathrm{Eu}$. Efforts continued to devise an optimum multiprocess procedure suitable for use in separating radioactive ${ }^{155} \mathrm{Eu}$ already produced by irradiation of stable ${ }^{154} \mathrm{Sm}$ in a high neutron flux reactor at the Institut Laue-Langevin in France and shipped to LANL (the 22-min ${ }^{155} \mathrm{Sm}$ neutron-capture product decays to ${ }^{155} \mathrm{Eu}$ ). This separation is extremely demanding because the highly radioactive ${ }^{155} \mathrm{Eu}$ must be isolated from about 20 times as much mass of samarium before a target can be prepared for DANCE measurements.

After all the procedures have been fully tested the radioactive ${ }^{155} \mathrm{Eu}$ will be separated. The same electroplating methods already used successfully to prepare stable Eu isotope targets will be used to prepare the ${ }^{155} \mathrm{Eu}$ target for DANCE. Discussions were held with LANL radiochemists in the Chemistry (C) Division about appropriate facilities at LANL for conducting the full-scale separation and purification of the radioactive targets. Three more multiprocess separations were developed that generated less chemical and radioactive waste, but they must still be adapted for processing hundred-milligram quantities. Until these separations can be successfully implemented at this scale, standard HPLC procedures will be used for separating and preparing radioactive ${ }^{155} \mathrm{Eu}, 2.6$-year ${ }^{147} \mathrm{Pm}$, and 1.9-year ${ }^{171} \mathrm{Tm}$ target materials.

Future directions beyond the preparation of radioactive lanthanide targets include closer collaboration with both LLNL and LANL to prepare actinide targets such as plutonium, americium, and curium. Also, the applicability of established and novel techniques will be evaluated for rapid separations of Am and $\mathrm{Cm}$ required in the irradiation of ${ }^{241} \mathrm{Am}$. Lastly, we will conduct a series of experiments aimed at enhancing current methods used to electrodeposit lanthanide and actinide targets on thin Ti and Be backings.

\section{INTRODUCTION}

The primary objective of this project is to measure neutron capture cross sections $\left(\sigma_{\mathrm{n}, \gamma}\right)$ for unstable nuclei using the newly developed Detector for Advanced Neutron Capture Experiments (DANCE) located at LANSCE. Accurate measurements of $\sigma_{\mathrm{n}, \gamma}$ for unstable nuclei have direct application to National Laboratory mission-critical programs such as Science Based Stockpile Stewardship, nuclear event attribution, and the Advanced Fuel Cycle Initiative. In addition, such information is invaluable to astrophysicists attempting to quantify the s- or "slow" process of nucleosynthesis. Since so few measurements of $\sigma_{\mathrm{n}, \gamma}$ for unstable nuclei exist, a priority list of $\sigma_{\mathrm{n}, \gamma}$ data requirements for several unstable nuclei was established in collaboration with scientists from several universities and national labs (known as the DANCE Collaboration). This list was 
generated based upon several factors including current experimental capabilities, availability of target material, importance to Laboratory mission-critical programs, and impact on basic science.

So far, we and our DANCE collaborators have identified Be as a suitable low-background target backing material, optimized an electrodeposition method for lanthanide (Ln) elements on thin Be foil, and fabricated three stable Eu targets of importance to radiochemical detector diagnostics $\left({ }^{151} \mathrm{Eu},{ }^{153} \mathrm{Eu}\right.$, and a 1:1 mixture of both). We have also begun preparations for measuring three unstable Ln nuclei, ${ }^{147} \mathrm{Pm},{ }^{155} \mathrm{Eu}$, and ${ }^{171} \mathrm{Tm}$, from the "priority" list of unstable isotopes that need to be investigated. Details of how the radioactive target material was produced are presented in last year's scientific report [1]. As part of the preparations it is necessary to develop capable procedures to separate the radioactive material from the stable Ln neighbor from which it was produced. This year we report significant progress in this area and present our final plans for preparing targets of the three radioactive lanthanide isotopes chosen.

Separating mixtures of lanthanides is not a trivial task. Elements in this group characteristically exhibit similar chemical behavior due to their like charge $\left(3^{+}\right)$and similar ionic radii. However, several lanthanides, including $\mathrm{Eu}$ and $\mathrm{Sm}$, can exist in oxidation states other than the predominant $3+$ state. In the past, this has been proven to be useful for separations [2]. Eu(III) is easier to reduce than Sm(III) [3,4]. The III/II reduction potentials for Eu and Sm are $-0.36 \mathrm{~V}$ and $-1.55 \mathrm{~V}$, respectively. This means by careful selection of a reductant, the redox characteristics of Eu may be exploited to enhance its separation from Sm. This combination of a reduction process as a first step followed by a chemical separation process as a second step is referred to here as multiprocess separation. Since a multiprocess separation method can enhance separation performance over traditional single process separation techniques and Tm and Pm are not redox sensitive, we have chosen to focus first on the development of a multiprocess method for separating the radioactive ${ }^{155} \mathrm{Eu}$ from the stable ${ }^{154} \mathrm{Sm}$ from which it was produced. Due to the required purity ( $>99 \%$ ) for DANCE targets and limited quantity of the radioactive material for making these targets (a few milligram quantities each), $\mathrm{Tm} / \mathrm{Er}$ and $\mathrm{Pm} / \mathrm{Nd}$ separations will be done using High-Performance Liquid Chromatography (HPLC).

Previously developed multiprocess methods for separating Eu from Sm all rely on the stabilization of $\mathrm{Eu}(\mathrm{II})$ as a sulfate precipitate during separation [5-32]. By forming $\mathrm{Eu}(\mathrm{II}) \mathrm{SO}_{4(\mathrm{~s})}$, the highly reducing conditions required to shift the redox equilibrium from $\mathrm{Eu}(\mathrm{III})$ to $\mathrm{Eu}$ (II) only have to be maintained to the point of precipitation. After this point the dissolution kinetics of $\mathrm{Eu}(\mathrm{II}) \mathrm{SO}_{4(\mathrm{~s})}$ stabilize the $\mathrm{Eu}(\mathrm{II})$. As a consequence, however, yield and purity (typically lower than $80 \%$ and $90 \%$, respectively) are sacrificed by leaving behind the amount of Eu required to exceed the solubility product and by co-precipitating contaminants with the sulfate solid. Subsequent re-dissolution and re-precipitation steps can in some cases be used to enhance purity, albeit at the price of lowering the overall yield [21]. In any case, re-precipitation cycles alone are not capable of attaining the level of purity $(>99 \%)$ required here. 
Our approach was to enhance yield and purity over existing multiprocess methods by avoiding the formation of a solid during separation. During our first year of funding (2003) we demonstrated the feasibility of this kind of approach by developing a high-purity multiprocess method capable of attaining $>99 \%$ purity Eu from excess (20:1 w:w) Sm [1]. The method coupled a Eu(III) reduction process using a $\mathrm{Zn}(\mathrm{Hg})$ Jones Reductor with a solvent extraction (SX) process that used thenoyltrifluoroacetone (TTA) in benzene. While we attained the level of purity required by the project in three sequential extractions, this success was offset by other issues related to the generation and disposal of radioactive mixed waste. This year we continued development work of multiprocess separation methods that could attain $>99 \%$ purity with a focus on also avoiding producing radioactive mixed waste in the process. This effort yielded three new multiprocess methods that generate less chemical and radioactive waste. Two methods, referred to here as Zn-SX and Zn-RP, respectively, couple a chemical reduction process using pure Zn metal to either SX using di-(2-ethylhexyl)orthophosphoric acid (HDEHP) in hexane or reversed phase (RP) chromatography using an HDEHP-based resin. The third method, referred to here as PSTAT-SX, combines an electrochemical reduction process using a potentiostat (PSTAT) and a glassy carbon (GC) working electrode with an SX separation using HDEHP in hexane.

The performance of each of these methods is presented here and compared to the multiprocess methods developed during the first year of funding. In addition, stable Eu targets we developed at Berkeley in 2003 were run at DANCE. These include isotopically enriched ${ }^{151} \mathrm{Eu}$ and ${ }^{153} \mathrm{Eu}$ as well as natural Eu (roughly 50:50 mixture of ${ }^{151} \mathrm{Eu}$ and ${ }^{153} \mathrm{Eu}$ ) targets. Members of the Berkeley Group traveled to Los Alamos to participate in the DANCE runs. Some of the preliminary data from these experiments are presented here.

\section{EXPERIMENTAL DETAILS}

\subsection{Tracers and Detection Methods}

Tracer solutions containing microcurie amounts of ${ }^{152} \mathrm{Eu}\left(\mathrm{t}_{1 / 2}=13.5\right.$ years $)$ and ${ }^{133} \mathrm{Ba}\left(\mathrm{t}_{1 / 2}=10.5\right.$ years) were used to investigate the performance of several newly proposed "high-purity" multiprocess separation methods. Studies of individual separation processes were carried out with and without an initial reduction process. ${ }^{133} \mathrm{Ba}$ (II) was used as an analog for $\mathrm{Ln}$ (II), while ${ }^{152} \mathrm{Eu}$ was used as an analog for both $\mathrm{Ln}(\mathrm{III})$ and $\mathrm{Ln}(\mathrm{II})$ behavior, depending on whether a reduction process was included in the experiment. Gamma peaks at $356 \mathrm{keV}$ and $121 \mathrm{keV}$ from the decay of ${ }^{133} \mathrm{Ba}$ and ${ }^{152} \mathrm{Eu}$, respectively, were analyzed using a high purity p-type germanium detector. The detector was calibrated for energy and efficiency using a commercial multinuclide standard. Counting times were varied to ensure that statistical errors were less than 5\% and typically less than $1 \%$. 


\subsection{Reductants for the First Step of the Multiprocess Method}

One new chemical and two new electrochemical reduction processes were tested this year for their ability to reduce $\mathrm{Eu}(\mathrm{III})$ and for their compatibility with various III/II metal cation separation processes. The reduction process will be the first step in the proposed multiprocess method. The new chemical reduction method utilizes excess amounts of acid cleaned, 30-mesh, pure $\mathrm{Zn}$ metal pellets. The reduction potential for $\mathrm{Zn}^{2+} / \mathrm{Zn}^{0}$ is $-0.763 \mathrm{~V}$ [4]. This potential lies between that of $\mathrm{Eu}^{3+} / \mathrm{Eu}^{2+}$ and $\mathrm{Sm}^{3+} / \mathrm{Sm}^{2+}$, allowing $\mathrm{Zn}^{0}$ to act as a selective reductant for $\mathrm{Eu}(\mathrm{III})$. A disadvantage of this technique is the addition of significant amounts of $\mathrm{Zn}^{2+}$ that eventually have to be separated from Eu. In addition, $\mathrm{Zn}^{0}$ is a strong reductant and is capable of reducing $\mathrm{H}^{+}$to $\mathrm{H}_{2(\mathrm{~g})}$ in acidic solutions [33]. To reduce competition from $\mathrm{H}^{+}, \mathrm{Zn}(\mathrm{Hg})$ amalgam is often used at the price of producing Hg-containing wastes. The combination of radioactive liquid wastes with $\mathrm{Hg}$ is designated a mixed waste and is subject to stricter disposal regulations and higher disposal costs. To avoid this, we attempted to use a pure $\mathrm{Zn}$ metal reductant by carefully selecting the solution $\mathrm{pH}$ so as to simultaneously minimize the $\mathrm{H}^{+}$competition for electrons at low $\mathrm{pH}$ and still avoid the formation of $\mathrm{Eu}(\mathrm{OH})_{2(\mathrm{~s})}$ at higher $\mathrm{pH}$.

The electrochemical reduction methods tested used either Pt or GC working electrodes with an EG\&G Instruments Potentiostat/Galvanostat model 283, a Pt counterelectrode, and a saturated $\mathrm{Ag} / \mathrm{AgCl}$ reference electrode. Both working electrodes are inert and so offer an effective means of controlling the redox state of Eu without adding chemical reductants to the system. However, the reduction potential of $\mathrm{Eu}(\mathrm{III}) / \mathrm{Eu}(\mathrm{II})$ lies close to the estimated lower end of the working range for both the Pt and GC electrodes [33,34]. Since this working range is greatly affected by the chemical composition of the solution, cyclic voltammetry (CV) was used in preliminary experiments to confirm that both electrodes could effectively reduce $\mathrm{Eu}(\mathrm{III})$ under the conditions expected during multiprocess separation. Cyclic voltammograms were generated for $10^{-3} \mathrm{M} \mathrm{Eu}$ solutions in 0.01-1.0 $\mathrm{M} \mathrm{HCl}$ at various scan rates and over various potential ranges (see below). A series of acid concentrations was also tested since low acid concentrations would benefit the separation process, while higher acid concentrations would benefit the reduction process.

\subsection{Separation Methods for the Second Step of the Multiprocess Method}

Initial experiments were conducted on several SX and RP chromatography separation techniques for potential use in the separation step of the multiprocess method. Two well-known 3+ organic complexants, HDEHP and TTA, were tested, either dissolved within one of a number of solvents or attached to a non-ionic substrate. In addition, tests were conducted on one proprietary RP resin known as LN2, available from Eichrom, Inc. The functional groups on the LN2 resin are thought to be similar in chemical character to those of HDEHP.

\subsubsection{Preliminary Solvent Extraction (SX) Experiments}

Solutions of 0.2 M HDEHP in hexane and $1 \mathrm{M}$ TTA in hexane, toluene, and benzene were tested for their ability to extract $\mathrm{Eu}(\mathrm{III})$ and $\mathrm{Ba}(\mathrm{II})$ from of $0.1 \mathrm{M} \mathrm{HCl}$. One $\mathrm{mL}$ of each organic phase 
and aqueous phase containing tracer were placed into a 4-mL test tube, vigorously shaken for 20 minutes, and then centrifuged for 3 minutes to sufficiently segregate the two phases. An autopipette, using a clean tip each time, was used to take a $0.75-\mathrm{mL}$ aliquot from both the organic and aqueous phases for counting. A $0.4-\mathrm{mL}$ aliquot containing the remainder of the organic phase was discarded prior to sampling the aqueous phase so as to minimize cross contamination.

\subsubsection{Preliminary Reversed Phase (RP) Experiments}

An RP resin which uses HDEHP-like functional groups was produced in-house in similar fashion to Horowitz et al. [35]. Briefly, $50 \mathrm{~g}$ of Amberlite XAD-7 resin was cleaned by first contacting it with $150 \mathrm{~mL}$ of deionized water for 30 minutes. The resin and water were then separated by vacuum filtration. Afterwards, roughly $150 \mathrm{~mL}$ of methanol was percolated through the resin. The HDEHP RP resin was produced by mixing $10 \mathrm{~g}$ of clean XAD-7 resin with $30 \mathrm{~mL}$ of methanol and $3.3 \mathrm{~mL}$ of 0.4 M HDEHP in methanol for 15 minutes. After mixing, the methanol was evaporated under vacuum using a heat lamp. Attempts to make a TTA-based RP resin by this method were unsuccessful.

Preliminary Eu and Ba sorption experiments were conducted on HDEHP and Eichrom, Inc. proprietary LN2 RP resins. Roughly $0.05-0.5$ g of HDEHP or LN2 RP resin that had been preequilibrated in excess acid of the appropriate concentration was weighed out and added to a 4$\mathrm{mL}$ test tube. Next, $1.25 \mathrm{~mL}$ of $0.01-1 \mathrm{M} \mathrm{HCl}$ or $\mathrm{HNO}_{3}$ was added to the test tube and shaken for 30 minutes. Mixtures were centrifuged and a 1-mL aliquot of the aqueous phase was collected for counting. To ensure material balance, a second 30-minute extraction was done using $6 \mathrm{M} \mathrm{HNO}_{3}$ to extract the remainder of the tracer still residing on the resin.

\subsection{Multiprocess Separations}

Based upon preliminary experiments, three new multiprocess methods for separating Eu from Sm were designed and tested. One method coupled electrochemical reduction with SX, while the other two methods coupled chemical reduction using $\mathrm{Zn}$ metal with either RP chromatography or SX. All methods were assessed for their ability to (1) efficiently extract Ln(III) from dilute acid and (2) do this preferentially over 2+ metal ions.

\subsubsection{Electrochemical Reduction: Solvent Extraction Multiprocess Method (PSTAT-SX)}

A multiprocess method termed PSTAT-SX that coupled electrochemical reduction using a GC working electrode with SX using HDEHP in hexane was developed and tested. The reduction apparatus was identical to that used during preliminary experiments. The performance of PSTAT-SX was assessed in the following manner. Seven $\mathrm{mL}$ of $0.1 \mathrm{M} \mathrm{HCl}$ containing Eu tracer was introduced into the potentiostat reactor and sparged with $\mathrm{N}_{2(\mathrm{~g})}$ for 15 minutes. A constant potential of $-0.52 \mathrm{~V}$ versus a Normal Hydrogen Electrode (NHE) was applied for 20 minutes. Afterwards, sparging was stopped for enough time to take an aliquot of the aqueous phase and add an equal volume of the organic phase to the reactor. For 25 minutes aliquots were 
periodically taken from the organic phase, after which time the organic and aqueous phases were separated and counted.

\subsubsection{Chemical Reduction: Reversed Phase Multiprocess Method (Zn-RP)}

A second multiprocess method developed and tested, termed Zn-RP, used Zn to first reduce Eu(III) and then HDEHP RP resin to separate Eu(II) from Sm(III). The performance of this method was judged using $\mathrm{Ln}(\mathrm{II})$ and $\mathrm{Ln}(\mathrm{III})$ tracers in a series of sorption experiments in the presence and absence of excess $\mathrm{Zn}$ reductant. Approximately $0.05 \mathrm{~g}$ of acid conditioned RP resin was placed in a 4-mL test tube containing $2 \mathrm{~mL}$ of $0.01-0.075 \mathrm{M} \mathrm{HNO}_{3}$ and approximately $0.2 \mathrm{~g}$ of acid-rinsed 30-mesh Zn metal. The mixture was shaken for 10 minutes, centrifuged, and aliquots taken for counting. Subsequent extractions using $6 \mathrm{M} \mathrm{HNO}_{3}$ were conducted to collect any tracer remaining on the RP resin and counted to assure material balance.

\subsubsection{Chemical Reduction: Solvent Extraction Multiprocess Method ( $\mathrm{Zn}-\mathrm{SX}$ )}

The third multiprocess method developed and tested this year combined the chemical reduction of Eu(III) using Zn metal and SX using HDEHP in hexane. The effectiveness of this method was assessed by a series of SX experiments in the presence and absence of the reductant. The reductant, roughly $0.2 \mathrm{~g}$ of $\mathrm{Zn}$, was placed into a test tube and equilibrated with $1.25 \mathrm{~mL}$ of tracer-containing 0.01-0.73 M nitric acid solution by shaking for 10 minutes. Then, $1.25 \mathrm{~mL}$ of 0.2 M HDEHP in hexane was added to all test tubes (both with and without Zn) and gently shaken for 20 minutes. Aliquots were periodically taken from both phases and counted.

\section{RESULTS AND DISCUSSION}

\subsection{Effectiveness of GC and Pt Working Electrodes for Reducing Eu(III) in Acidic Solutions}

In theory, multiprocess methods that utilize electrochemical means to reduce $\mathrm{Eu}(\mathrm{III})$ are preferable over chemical reduction processes that would contaminate the system with $\mathrm{Zn}$. However, the ability of the electrochemical process to effectively reduce Eu(III) in acid solutions had to be verified. To do this, cyclic voltammetry (CV) was used to determine the lower working range of both GC and Pt working electrodes. To be effective, the reduction potential for $\mathrm{Eu}(\mathrm{III}) / \mathrm{Eu}(\mathrm{II})$ has to fall within the working range of the working electrode under specified conditions. A range of acid solutions were also tested since low acid concentrations would benefit the separation process, while higher acid concentrations would reduce the working range of the working electrodes. For a reversible one-electron transfer reaction, a CV scan across the reduction potential of a redox couple produces two waves of changing current over the baseline reading. These waves are caused by a depletion of the reductant or oxidant near the electrode [36]. In all scans using a Pt working electrode, no clear Eu reduction/oxidation waves were observed. From this result we concluded that the working range of Pt was not low enough to reduce $\mathrm{Eu}(\mathrm{III})$ under the specified experimental conditions. However, using a GC working electrode, Eu reduction/oxidation waves were observed for acid concentrations $\geq 0.1 \mathrm{M}$ (Figure 
1). The location and character of the anode and cathode half-peak potentials suggested the reaction was reversible and transferred a single electron, consistent with $\mathrm{Eu}(\mathrm{III})$ reduction to $\mathrm{Eu}(\mathrm{II})$. In addition, the reduction potential for the $\mathrm{Eu}(\mathrm{III}) / \mathrm{Eu}(\mathrm{II})$ couple was measured to be $-0.350 \mathrm{~V}$, comparable to previously published values [4]. Therefore we conclude that the GC working electrode could effectively reduce $\mathrm{Eu}(\mathrm{III})$ in $\geq 0.1 \mathrm{M} \mathrm{HCl}$.

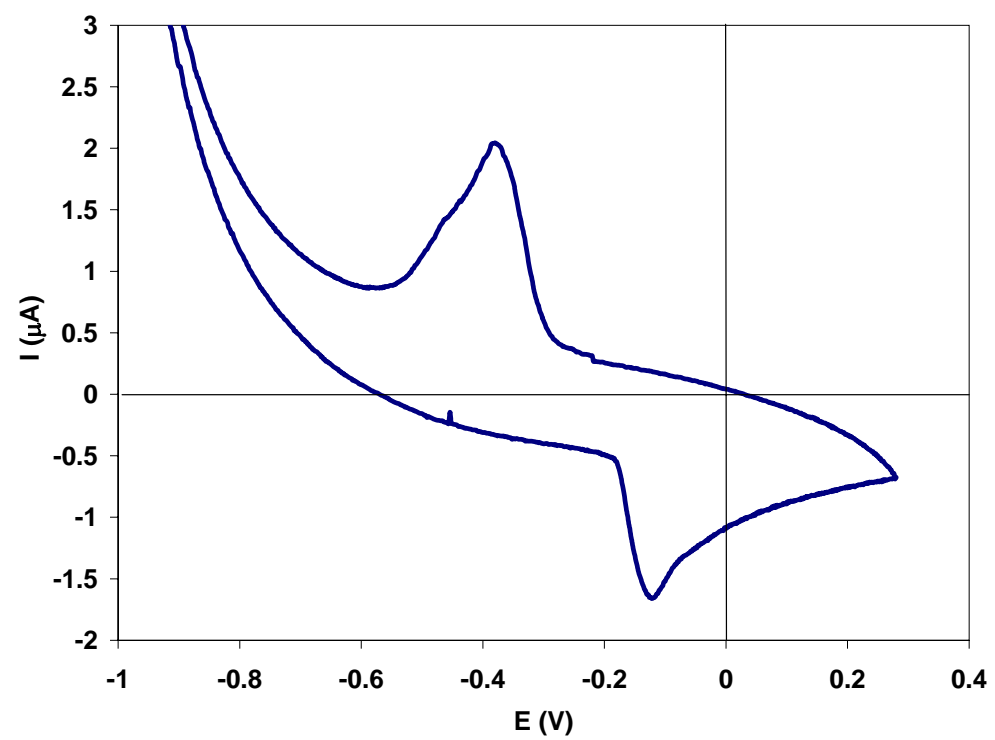

Figure 1. Cyclic voltammogram of $10^{-3} \mathrm{M}$ Eu solution in $0.1 \mathrm{M} \mathrm{HCl}$. The figure represents an average of three scans from -1 to $+0.27 \mathrm{~V}$ (Vs. NHE) at a scan rate of 20 $\mathrm{mV} / \mathrm{s}$, using a GC working electrode, a Pt counterelectrode, and a saturated $\mathrm{Ag} / \mathrm{AgCl}$ reference electrode.

\subsection{Comparison of Eu(III) and Ba(II) Extractions Using Various SX Methods}

Prospective separation methods for use in a multiprocess technique have to effectively extract Ln(III) from dilute acid and do so with great preference over that of 2+ metal cations. Four solutions, 0.2 M HDEHP in hexane and 1 M TTA in hexane, toluene, and benzene, were tested for their ability to extract $\mathrm{Eu}(\mathrm{III})$ in the presence of $\mathrm{Ba}(\mathrm{II})$ from $0.1 \mathrm{M} \mathrm{HCl}$. The results of these tests are shown in Figure 2 and include previously published results [1] for TTA in benzene for comparison purposes. The distribution ratio (D) is equal to the ratio of the concentration of the respective tracer in the organic phase to that in the aqueous phase. The distribution ratio for $\mathrm{Ba}$ (II) (identified in the figure as D-II) for each solvent is indicated with open bars. Values for the separation factor (SF), equal to the ratio of $\mathrm{D}$ for $\mathrm{Eu}(\mathrm{III})$ to that for $\mathrm{Ba}(\mathrm{II})$, for each of the solvents is shown in the shaded bars. Using the following equations, it is possible to estimate maximum and minimum design values for D-II $\left(\mathrm{D}_{\mathrm{II}}{ }^{*}\right)$ and SF $\left(\mathrm{SF}^{*}\right)$, respectively, based upon minimum purity and yield criteria. Assuming a minimum yield for $\mathrm{Eu}(\mathrm{II})$ of $80 \%$, $\mathrm{D}_{\mathrm{II}}{ }^{*}$ was estimated to be $\leq 0.08$ after 3 extraction cycles from the following equation: 


$$
\mathrm{Y}_{\mathrm{n}}=\left(\frac{1}{1+\mathrm{D}_{\mathrm{II}}^{*}}\right)^{\mathrm{n}}
$$

where $Y_{n}$ is the yield after $n$ SX cycles. The purity of Eu after $n$ extractions $P_{n}$ is given by the equation

$$
\mathrm{P}_{\mathrm{n}}=\left[1+\left(\frac{1+\mathrm{D}_{\mathrm{II}}^{*}}{1+\mathrm{SF}^{*} \mathrm{D}_{\mathrm{II}}^{*}}\right)^{\mathrm{n}} \cdot \mathrm{R}\right]^{-1}
$$

where $\mathrm{R}$ is the ratio of the initial weights of $\mathrm{Sm}$ and $\mathrm{Eu}$. Using the estimated value for $\mathrm{D}_{\mathrm{II}}{ }^{*}$ and assuming $\mathrm{P}_{3} \geq 99 \%$, SF ${ }^{*}$ was calculated to be $\geq 161$. The relative location of design values for $\mathrm{D}$ II and SF are shown in Figure 2 as solid and dashed lines, respectively. Based upon the design criteria, further study was justified for HDEHP in hexane and TTA in benzene. TTA in benzene was studied in 2003 [1] but rejected as an extraction system because it generates more hazardous waste. As shown in the figure, HDEHP in hexane also has a lower D-II and a greater SF than TTA in benzene, making it more suitable as an extraction system.

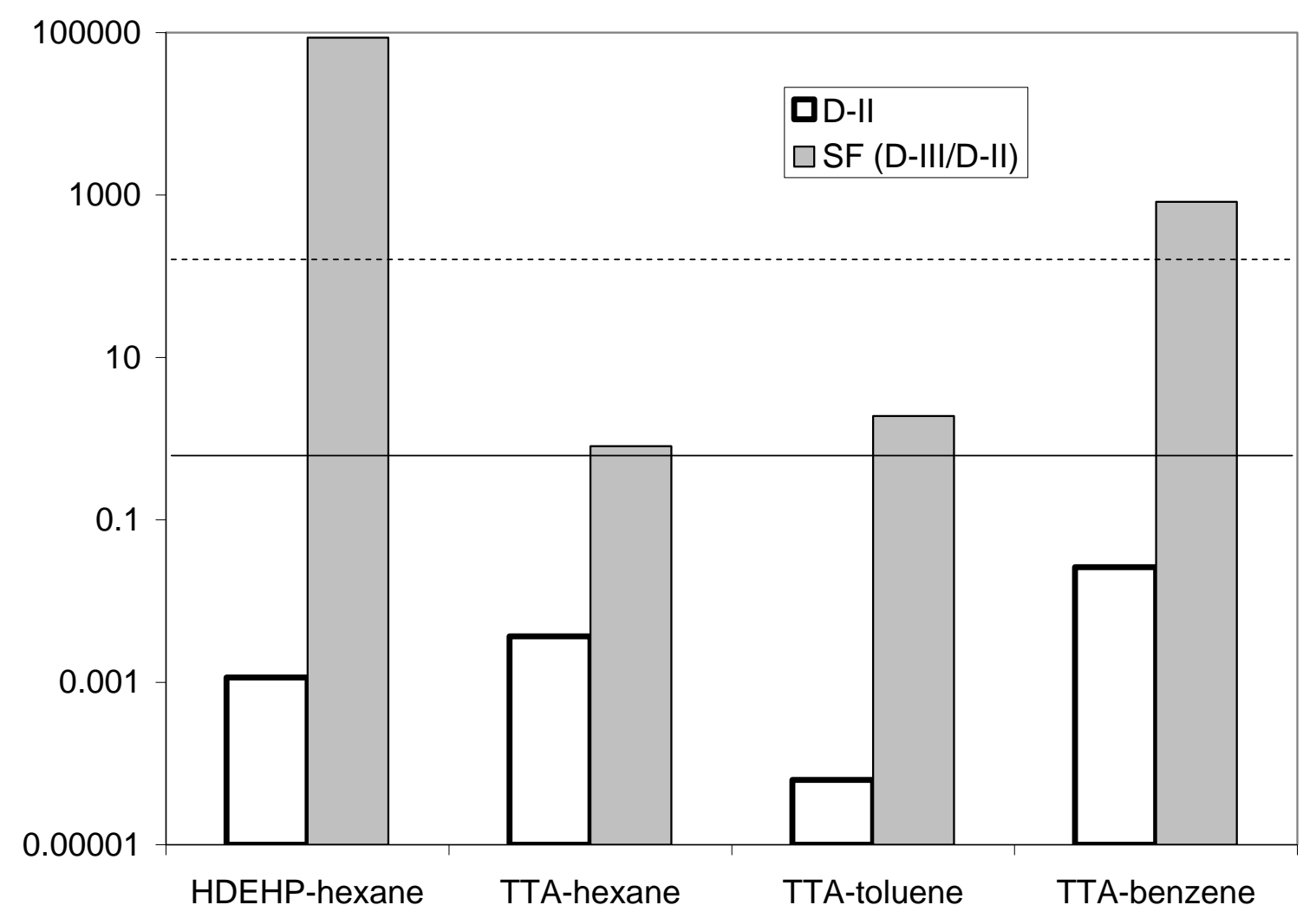

Figure 2. Relative performance and compatibility of various SX techniques for use in a multiprocess $\mathrm{Eu}(\mathrm{II}) / \mathrm{Sm}(\mathrm{III})$ separation. Open bars show the value of the distribution ratio, D-II, for Ba. Shaded bars represent the separation factor, SF (equal to D-III/D-II), for each of the solvents tested. The meaning of the solid and dashed lines is discussed in the text. 


\subsection{Sorption of Eu(III) and Ba(II) on RP HDEHP and LN2 Resins from Acidic Solutions}

Typically, HDEHP RP chromatography of Ln(III) ions is performed from dilute $\mathrm{HNO}_{3}$ [37]. However, since we planned to integrate SX with a reduction process, we attempted to use $\mathrm{HCl}$ instead of $\mathrm{HNO}_{3}$ to avoid an acid that might re-oxidize Eu(II) to Eu(III). Preliminary SX experiments using extractants with functional groups similar to those present on the surface of the in-house RP resin suggested such a substitution was possible.

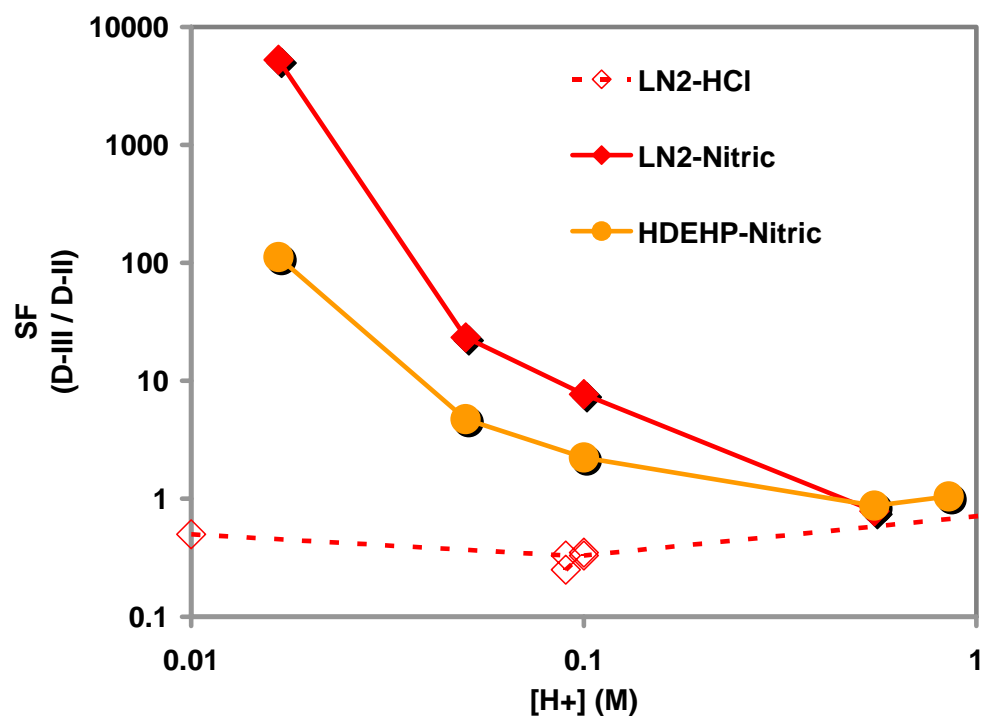

Figure 3. Acid compatibility of Ln(III) phosphate-type RP resins.

Results of Eu and Ba sorption experiments on RP resins are shown in Figure 3. These results indicate that the $\mathrm{RP}$ resin in $\mathrm{HCl}$ (shown in the dashed line) did not significantly favor the extraction of $\mathrm{Ln}\left(\mathrm{III}\right.$ ) over $\mathrm{Ln}(\mathrm{II})$. Identical experiments in $\mathrm{HNO}_{3}$ (shown in solid lines) seemed to indicate that phosphate-type resins such as HDEHP and LN2 are incompatible with HCl. This result came as a surprise, because HDEHP in hexane is capable of extracting $\mathrm{Ln}(\mathrm{III})$ out of $\mathrm{HCl}$ in SX experiments. The difference in complexation between the two anions might be a possible explanation for this unexpected behavior. Chloride ions are known to form stronger complexes with metal cations in aqueous solutions than nitrate ions. This difference in complexation might have a different effect on the interaction between resin and mobile phase than it does on the interaction between aqueous and organic phase in solvent extraction experiments. In addition, HDEHP attached to a resin should bind Ln(III) less strongly than if dissolved in solvent due simply to steric interferences. These two issues together may cause Ln(III) to favor complexation with $\mathrm{Cl}^{-}$ions in $\mathrm{HCl}$ rather than partition to the resin over the acid concentrations tested here. 


\subsection{PSTAT-SX}

A multiprocess separation (PSTAT-SX), which coupled electrochemical reduction using a GC working electrode and SX using HDEHP in hexane, was tested for its ability to separate Eu(II) from Sm(III). The average D for Eu while the reduction potential was applied was $\sim 41$. Based upon results from preliminary experiments (D measured for Eu(III) in HDEHP in hexane was $\sim 100$ without the presence of a reductant), this represents an effective SF of 2.4. Since single process separation methods typically produce SF's just greater than unity [3], a multiprocess method that obtains a SF much greater than this indicates that separation was enhanced by including a reduction step. While these results represent only a modest enhancement over traditional methods that do not reduce Eu prior to separation, this does validate the proposed multiprocess approach.

\subsection{Zn-RP}

A series of experiments was conducted to test the compatibility and performance of a $\mathrm{Zn}$ reductant and HDEHP RP resin as a multiprocess method for separating Eu(II) from Sm(III). Results from these tests (presented in Figure 4) show that the multiprocess combination was not effective at separating the two lanthanides. Interestingly, the distribution ratio (D) of Eu between the resin and solution was greatest for all acid concentrations when Zn was present, suggesting that the uptake of Eu(II) by the resin was greater than Eu(III). This is initially not intuitive since $\mathrm{Eu}(\mathrm{II})$ should have much lower $\mathrm{D}$ values than $\mathrm{Eu}(\mathrm{III})$ at lower acid concentrations. We interpret these results to mean $\mathrm{Zn}$ could not effectively reduce $\mathrm{Eu}(\mathrm{III})$ in $\mathrm{HNO}_{3}$ due to competition with $\mathrm{H}^{+}$ions. Instead, this reduction of $\mathrm{H}^{+}$ions by $\mathrm{Zn}$ likely caused $\left[\mathrm{H}^{+}\right]$to decrease, thereby enhancing the sorption of Eu(III) on the RP resins.

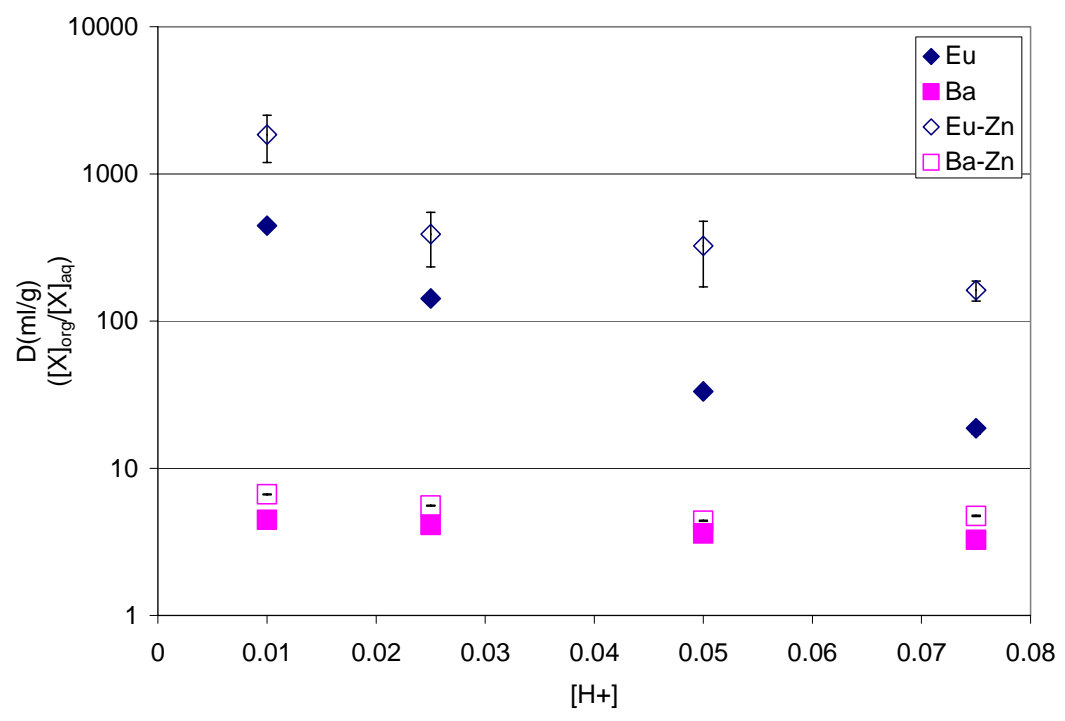

Figure 4. Distribution ratio (D) of Eu and $\mathrm{Ba}$ on $\mathrm{HDEHP} \mathrm{RP}$ resin in $\mathrm{HNO}_{3}$ in the presence and absence of $Z n$ reductant. 


\subsection{Zn-SX}

The $\mathrm{Zn}-\mathrm{SX}$ method was tested in a series of test tube experiments containing $\mathrm{Ln}^{2+}$ and $\mathrm{Ln}^{3+}$ tracers in 0.01-0.73 $\mathrm{M} \mathrm{HNO}_{3}$ in the presence of an equal volume of $0.2 \mathrm{M}$ HDEHP in hexane and excess Zn. The results of these tests are presented in Figure 5. The SF for Eu is the ratio of D's in Figure 5 for Eu without and with reductant added, respectively. As $\left[\mathrm{H}^{+}\right]$decreased, the SF increased to a maximum value of 16.5 in $0.01 \mathrm{M} \mathrm{HNO}_{3}$. However, this result was not sufficient to meet the minimum SF design criteria (161) set forth by this project (see the discussion in Section 4.2). At the highest $\left[\mathrm{H}^{+}\right](0.73 \mathrm{M})$, D for the Eu system containing reductant was greater than that for the system with no reductant. This effect is probably caused by competition between $\mathrm{H}^{+}$ions and $\mathrm{Eu}^{3+}$ for electrons, as described in Section 4.5. This competition is greatest at higher $\left[\mathrm{H}^{+}\right]$so it may not have been observable at lower $\left[\mathrm{H}^{+}\right]$.

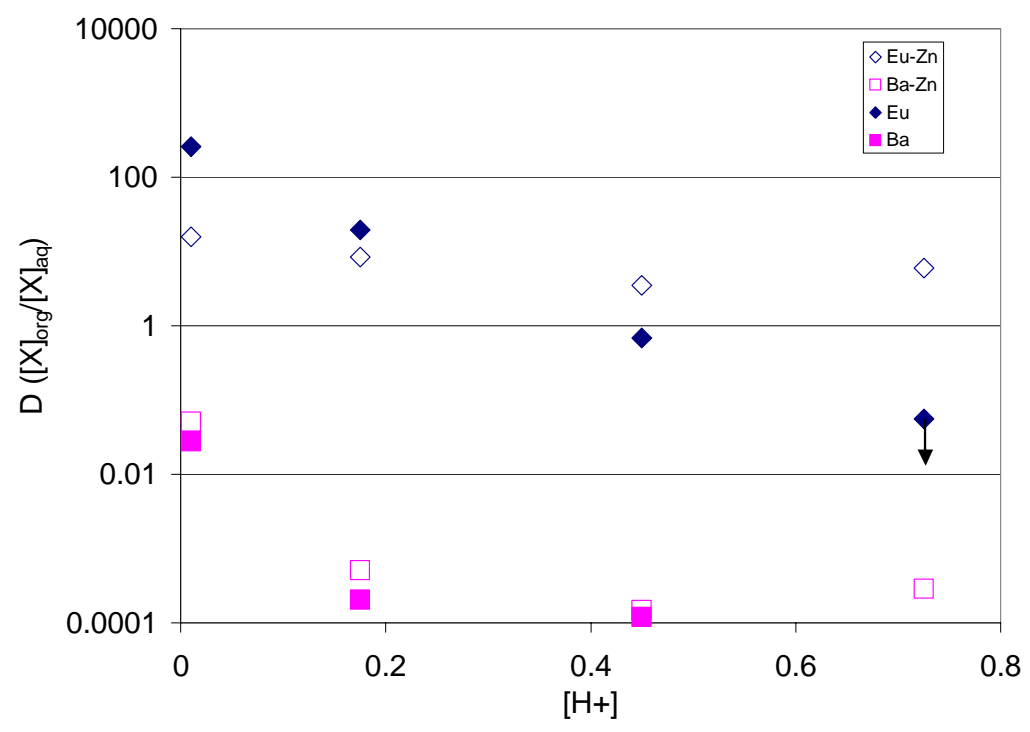

Figure 5. Distribution ratios (D) for Eu and Ba between 0.2 M HDEHP in hexane and $\mathrm{HNO}_{3}$ with and without the addition of $\mathrm{Zn}$ reductant. Down arrow indicates the data point represents a maximum possible value for $D$ based upon limited counting statistics. Recall that SF (not shown in the figure) is defined as the ratio of $D$ without and with $\mathrm{Zn}$ reductant.

\subsection{Assessment of Multiprocess Separation Methods}

Our experiments show that mixtures of Eu and Sm can be effectively separated by a multiprocess method that combines an initial reduction step from $\mathrm{Eu}(\mathrm{III})$ to $\mathrm{Eu}(\mathrm{II})$ followed by chemical separation of Eu(II) and Sm(III). Unlike previously developed multiprocess methods, this study has focused on the design and testing of several such techniques that do not rely on the stabilization of Eu(II) as a sulfate precipitate during separation. The success of these newly designed methods varied (see Figure 6) but the combination of a $\mathrm{Zn}(\mathrm{Hg}$ ) reductant with SX using TTA in benzene performed best. Based on the experimentally determined SF of this system, it is 
capable of obtaining theoretical Eu purity and yield of 99.8\% and 92\%, respectively, from a 20:1 (w:w) mixture of Sm and Eu after three extraction cycles. Although these results illustrate the capability of multiprocess methods, the best method generates waste containing benzene and $\mathrm{Hg}$. Methods that eliminated the use of these chemicals did not meet the design criteria.

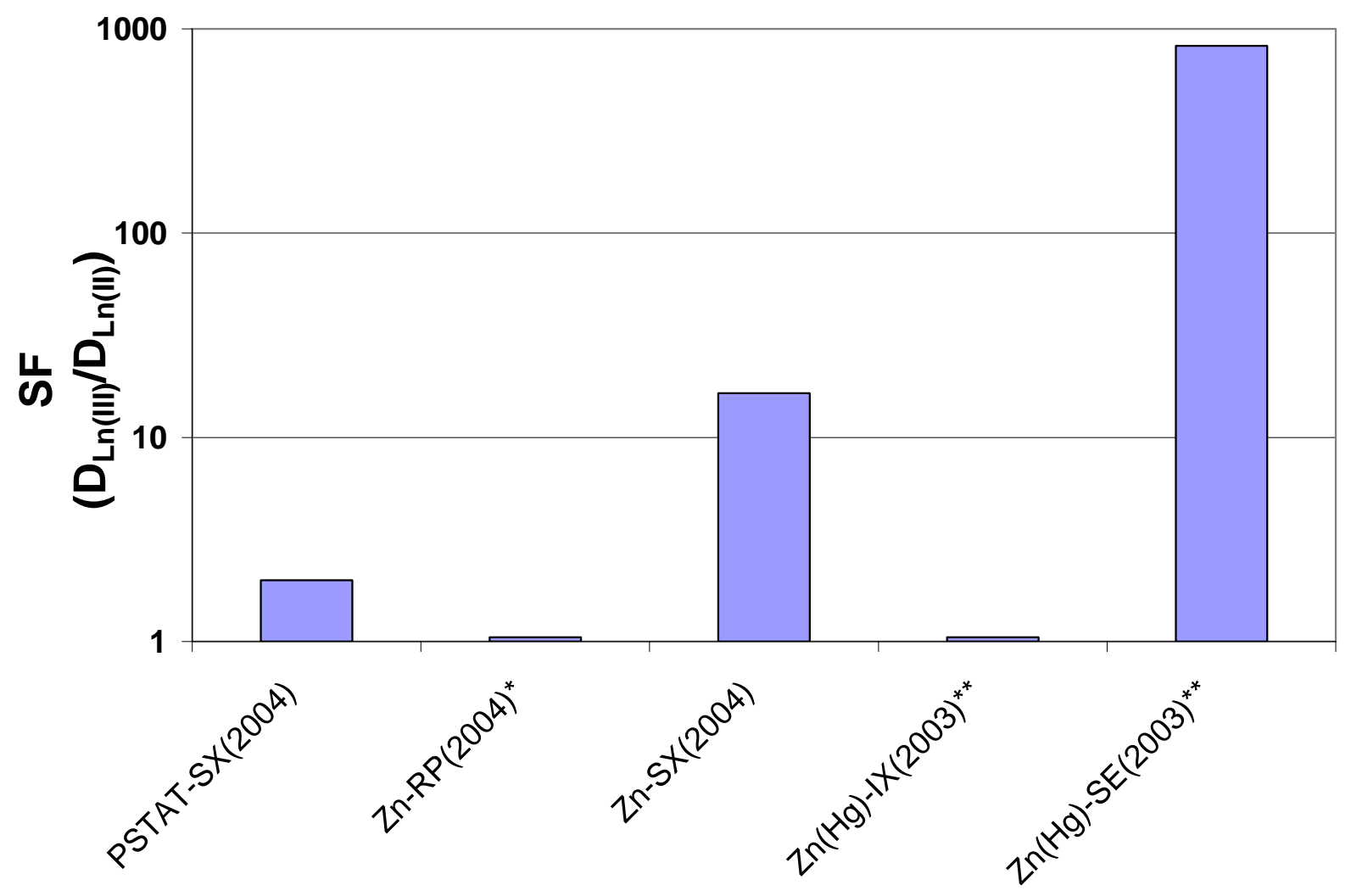

Figure 6. Comparison of all high-purity multiprocess methods designed and tested during the first year (indicated by "2003") and second (indicated by "2004") year of funding. ${ }^{*}$ The SF for $Z n-R P$ was calculated as the ratio of $K_{D-L n(I I I)} / K_{D-L n(I I) .}{ }^{* *}$ Details of these experiments can be found in last year's scientific report [1].

\section{PRELIMINARY $\sigma(n, \gamma)$ DATA FOR STABLE ${ }^{151}$ Eu AND ${ }^{153}$ Eu ISOTOPES}

This year we traveled to Los Alamos several times to participate in the measurement of neutron capture cross sections at the DANCE detector at the LANSCE facility. LANSCE is an accelerator-based facility capable of producing high-fluence beams of neutrons in the low keV region and is uniquely suited for measuring neutron capture cross sections of interest to stockpile stewardship based science. At the facility, $\mathrm{H}^{-}$and $\mathrm{H}^{+}$particles are produced within two separate high-voltage domes, mixed, and accelerated initially by a drift-tube linear accelerator (LINAC) to $100 \mathrm{MeV}$ and then further accelerated to $800 \mathrm{MeV}$ using a side-coupled cavity LINAC. The $\mathrm{H}^{-}$are separated from the $\mathrm{H}^{+}$ions and sent to the Proton Storage Ring, where macropulses (750 $\mathrm{ms})$ of these particles are converted to micropulse $(0.13 \mu \mathrm{s})$ bursts of intense $\mathrm{H}^{+}$ions. These 
protons are subsequently sent to the Lujan center for producing neutrons suitable for a variety of precise time of flight (TOF) experiments. At an incident energy of $800 \mathrm{MeV}$, approximately 20 neutrons are produced via spallation for every proton that hits the tungsten metal production target. This produces neutrons too high in energy for most applications, so prior to delivering beam to the 16 flight paths at the Lujan Center, the energies of the neutrons can be decreased by interaction with water, a light, neutron-scattering material.

The DANCE is located at flight path 14 at the LANSCE facility. A schematic of DANCE is shown in Figure 7. It consists of a shell of $160 \mathrm{BaF}_{2}$ detectors in nearly $4 \pi$ geometry around the beam line and was designed to have high efficiency for $\gamma$-ray detection, good neutron energy resolution, and low background with respect to neutrons. The $\mathrm{BaF}_{2}$ crystal detector material was chosen for its high efficiency, energy resolution, and the relatively fast recovery that is needed to prevent pileup of decay events between the rapid beam pulses to the target. In addition, DANCE is capable of a timing resolution of $135 \mathrm{~ns}$ (FWHM) which allows for accurate time of flight estimates of incident neutron energies.

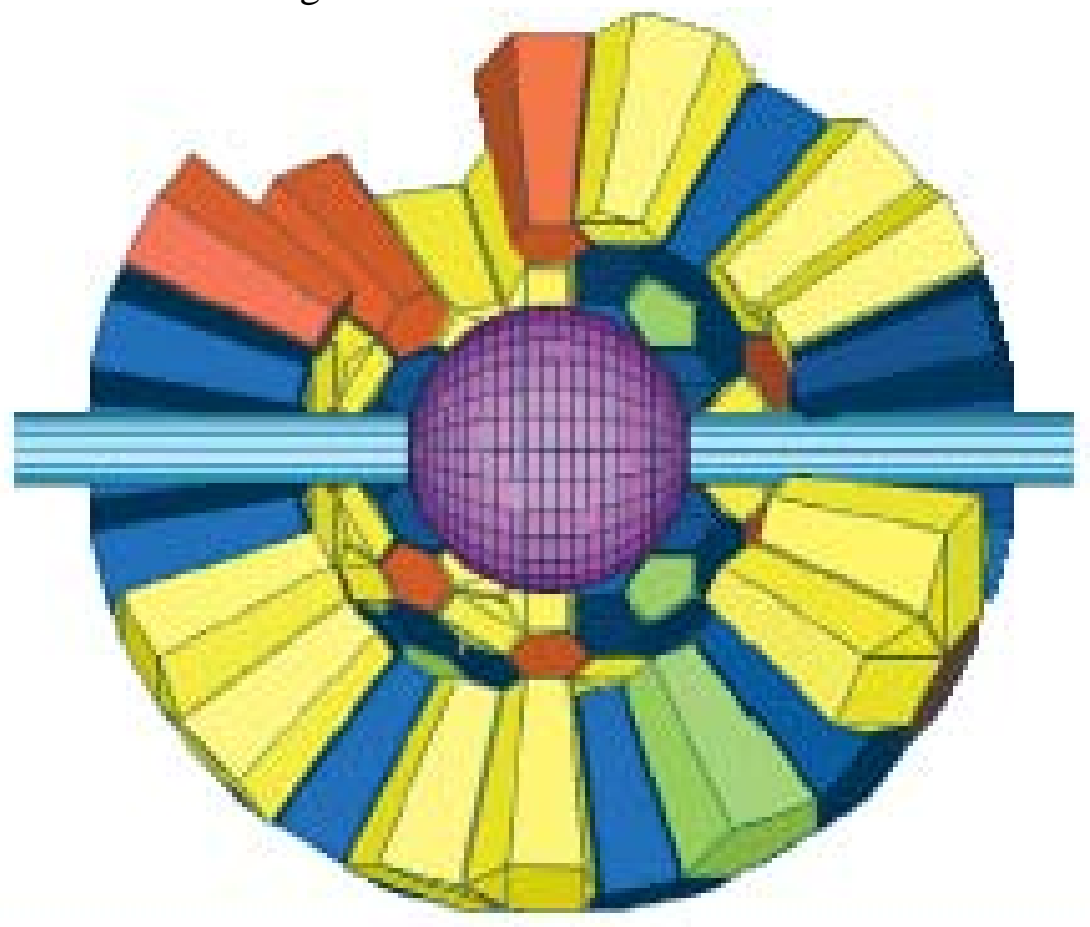

Figure 7. Schematic of the DANCE array. Each color represents a different shape of the crystal array required for $4 \pi$ geometry around the target.

During the runs at which scientists from Berkeley were present, data were collected using a variety of targets, but most importantly using the two stable Eu targets that were produced at Berkeley. These targets consisted of isotopically separated ${ }^{151} \mathrm{Eu}$ and ${ }^{153} \mathrm{Eu}$, electrodeposited to a thickness of $\sim 1 \mathrm{mg} \mathrm{cm}^{-2}$ on a 0.5 -mil Be backing (see [1] for details). They were irradiated along 
with Be blanks at LANSCE during early 2004. Data from segmented and continuous mode runs were collected for both isotopes [1]. The data from these experiments are still being processed and the necessary analysis modules are still under development at LANL. However, preliminary neutron energy and time of flight histograms, uncorrected for Doppler shift and background, are available. For illustration purposes, neutron TOF and energy histograms for the reaction ${ }^{151} \mathrm{Eu}(\mathrm{n}, \gamma){ }^{152} \mathrm{Eu}$ are shown in Figures 8a and 8b, respectively.

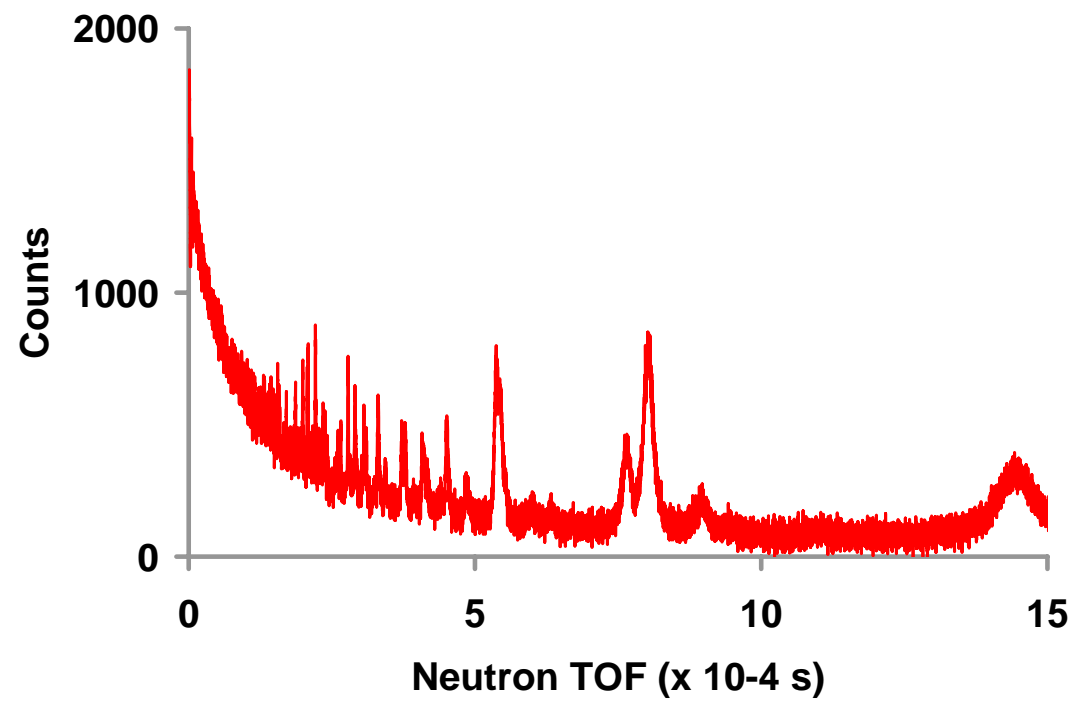

(a)

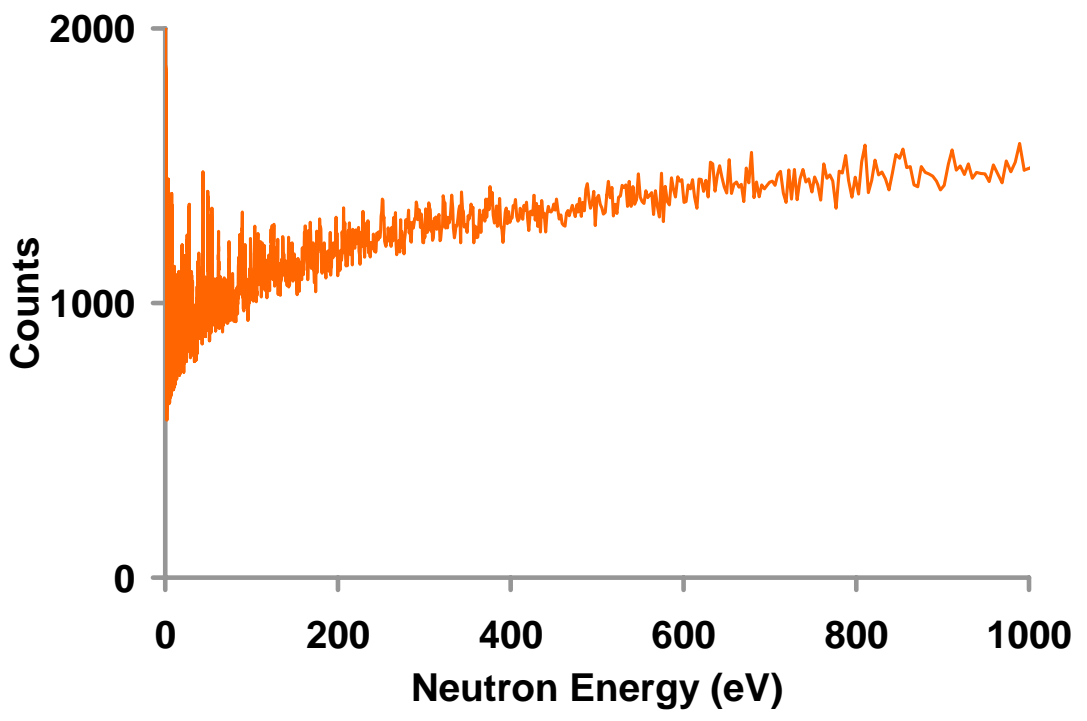

(b)

Figure 8. Neutron time of flight (a) and neutron energy (b) histograms for DANCE run \#1155. The target was $0.839 \mathrm{mg} \mathrm{cm}{ }^{-2}{ }^{151} \mathrm{Eu}$ on a 0.5-mil Be backing. The data was acquired in segmented run mode [1] with a looking time of $2 \mathrm{~ms}$ and total run time of $105 \mathrm{~min}$. 


\section{PERSONNEL}

The small subcontract with the Lawrence Berkeley National Laboratory (LBNL) Heavy Element Nuclear and Radiochemistry Group was continued in 2004 and renewed for the coming year (2005) to support staff scientist Dr. Ralf Sudowe (20\% time). His responsibilities include helping to train and supervise postdocs and students in laboratory procedures and safety, and conduct aspects of research associated with electroplating, lanthanide and actinide separations chemistry, and various radioanalytical measurements.

Dr. Schwantes left at the end of June 2004 to take a position at LANL with the Chemistry Division section performing experiments at DANCE. He intends to continue close collaboration with our Berkeley group and return in the near future to finish some electrodeposition experiments here that are needed prior to completing a paper for submission.

Dr. Schwantes' move to LANL was a great loss to us, but signaled our success in "fulfilling the goal of the SSAAP to help to train the next generation of scientists with training relevant to national security and stewardship science to carry out programs at the NNSA laboratories"! After conducting a national search and several interviews, C. M. Folden III was selected to fill the postdoctoral position. He is uniquely qualified and joined the project on November 5, 2005 after completing the requirements for his Ph.D. Additional students (1-2) will also be added to the project.

\section{FUTURE RESEARCH}

\subsection{HPLC Separations}

The three new multiprocess separations developed generated less chemical and radioactive waste, but they must still be adapted for processing hundred-milligram quantities. Until these separations can be successfully implemented at this scale, a single process method will be used for separating radioactive ${ }^{155} \mathrm{Eu}$ from stable ${ }^{154} \mathrm{Sm}$. A single process method will also be required to separate the radioactive target material ${ }^{171} \mathrm{Tm}$ and ${ }^{147} \mathrm{Pm}$ from the stable isotopes from which they were produced $\left({ }^{170} \mathrm{Er}\right.$ and ${ }^{146} \mathrm{Nd}$, respectively). A recent review of numerous single process methods [3] utilizing SX, ion exchange (IX) chromatography, and RP chromatography, showed that IX chromatography using cation exchange resin and an eluant containing alphahydroxyisobutyric acid $(\alpha-\mathrm{HIB})$ provides a feasible method for separating individual lanthanides. In addition, the resolution and speed of this technique can be greatly enhanced by employing high-performance liquid chromatography (HPLC) [37]. We plan to employ this technique for separating radioactive ${ }^{147} \mathrm{Pm},{ }^{155} \mathrm{Eu}$, and ${ }^{171} \mathrm{Tm}$ target material for DANCE experiments. Results from a number of previous experiments that have used IX HPLC and $\alpha$-HIB eluant to separate lanthanides [38-46] provide us with a basis from which to optimize the method for our particular purpose. We hope to have the first of these radioactive lanthanide targets ready by April 2005. 


\subsection{Separations Chemistry and Preparation of Actinide Targets}

One of our areas of interest in the next year is the neutron capture cross section of ${ }^{241} \mathrm{Am}$. Neutron capture in this nuclide produces both ground-state and isomeric ${ }^{242} \mathrm{Am}$, and we hope to investigate the cross section of both reactions. Plans are being made at LANL to irradiate ${ }^{241} \mathrm{Am}$ at the reactor facility in Karlsruhe, Germany and perform subsequent analysis of the sample. Ground-state ${ }^{242 \mathrm{~g}} \mathrm{Am}$ decays rapidly $\left(\mathrm{t}_{1 / 2}=16.0 \mathrm{~h}\right)$ to ${ }^{242} \mathrm{Cm}$, which will be separated and assayed to determine the ${ }^{241} \mathrm{Am}(\mathrm{n}, \gamma){ }^{242 \mathrm{~g}} \mathrm{Am}$ cross section. A new, rapid separation technique will need to be developed to minimize the growth of ${ }^{242} \mathrm{Cm}$ once the separation begins. ${ }^{242 \mathrm{~m}} \mathrm{Am}\left(\mathrm{t}_{1 / 2}=141 \mathrm{y}\right)$ decays by isomeric transition to ${ }^{242 \mathrm{~g}} \mathrm{Am}$ which will decay to ${ }^{242} \mathrm{Cm}$ as before. Once the first separation is complete, the sample can be stored until sufficient ${ }^{242} \mathrm{Cm}$ grows in (likely six months or more). The curium will be separated a second time and assayed to determine the ${ }^{241} \mathrm{Am}(\mathrm{n}, \gamma){ }^{242 \mathrm{~m}} \mathrm{Am}$ cross section. After a thorough literature search, the applicability of established and novel techniques will be evaluated for rapid separations of Am and Cm.

\section{3. $\quad$ Enhanced Electroplating Methods}

In the coming year, we will conduct a series of experiments aimed at enhancing current methods used to electrodeposit lanthanide and actinide targets on thin $\mathrm{Ti}$ and Be backings. Deposition thickness and homogeneity as a function of voltage and plating time and will be determined. In preparation for this work, a new computer-controlled power supply (Stanford Research Systems model PS310) has been purchased. This new power supply has the capability of stepping the voltage during deposition, a process that may enhance the quality of the final deposit. The results of these electrodeposition experiments will be combined with those conducted during the previous year and a manuscript will be submitted for publication. In addition, we will investigate deposition on ultra-thin exotic backing materials.

\subsection{Data Analysis of ${ }^{155}$ Eu}

This year we plan to begin analysis of neutron-capture data collected when a target of radioactive ${ }^{155} \mathrm{Eu}$ is irradiated at the LANSCE facility. Beginning in February 2005, a new DANCE data format will be used which will more accurately represent the waveforms produced by the DANCE detectors. This new data format has been finalized by the DANCE group and the "preprocessing” codes (also called Stage One) used to make this data suitable for thorough analysis are in the later stages of development. Previously, Stage One was done under the framework of the MIDAS system; under the new system this will be replaced by a proprietary algorithm. The Stage One data will be uploaded to Berkeley for analysis. In preparation, a new high-performance workstation with redundant storage has been purchased by our group and is ready for use. We have also been in extensive contact with the DANCE group to discuss details of the final analysis. We expect that the ${ }^{155} \mathrm{Eu}$ target will be irradiated in April 2005. 


\section{REFERENCES}

[1] Schwantes, J. M.; Hoffman, D. C.; Sudowe, R.; Nitsche, H.; Ann. Tech. Rep. 2003, DOE/NA/00075-1, 45 pages, available at http://www.osti.gov/servlets/purl/820513cno3OK/webviewable/.

[2] Preston, J. S.; du Preez, A. C.; J. Chem. Tech. Biotech. 1996, 65, 93.

[3] Stevenson, P. C.; Nervik, W. E.; The Radiochemistry of the Rare Earths Scandium, Yttrium, and Actinium; USAEC: Livermore, Vol. 3020, 1961.

[4] Lide, D. R.; CRC Handbook of Chemistry and Physics; CRC Press, Ann Arbor, 1994.

[5] McCoy, H. N.; J. Am. Chem. Soc. 1937, 59, 1131.

[6] Stone, H. W.; Hume, D. N.; Ind. Eng. Chem. 1939, 11, 598.

[7] Marsh, J. K.; J. Chem. Soc. 1943, 531.

[8] Hillebrand, W. F.; Lundell, G. E. F.; Hoffman, J. I.; Applied Inorganic Analysis; Wiley: New York, 1953.

[9] Morais, C. A.; Ciminelli, V. S. T.; Hydrometallurgy 1998, 49, 167.

[10] Morais, C. A.; Ciminelli, V. S. T.; Hydrometallurgy 2001, 60, 247.

[11] Yntema, L. F.; J. Am. Chem. Soc. 1930, 52, 2782.

[12] Onstott, E. I.; J. Am. Chem. Soc. 1955, 77, 2129.

[13] Lu, D.; Miao; Wu, Y. L.; Tung, C. P.; In Separation Processes in Hydrometallurgy; Davies, G. A., Ed.; Ellis Horwood: London, 1987, pp 428-436.

[14] Atanasyants, A. G.; Sen'kin, Y. A.; Ah. Prikl. Khim. 1990, 63, 2062.

[15] Atanasyants, A. G.; Sergin, A. N.; Danilov, A. V.; Kochetkov, V. L.; Zh. Prikl. Khim. 1990, 63, 2066.

[16] Hirai, T.; Komasawa, I.; J. Chem. Eng. Jpn. 1992, 25, 644.

[17] Hung, T. M.; Lee, C. J.; J. Appl. Electrochem. 1992, 22, 865.

[18] Atanasyants, A. G.; Seryogin, A. N.; Hydrometallurgy 1995, 37, 367.

[19] Kong, F. Q.; Yu, Z. Y.; Wang, B. H.; Qin, M.; Miao, S. H.; Zhao, B.; J. Rare Earth 1995, $13,170$.

[20] Melnik, M. I.; Karelin, E. A.; Filimonov, V. T.; Radiochemistry 1995, 37, 156.

[21] Preston, J. S.; du Preez, A. C.; Cole, P. M.; Fox, M. H.; Hydrometallurgy 1996, 42, 131.

[22] Yorukoglu, A.; Girgin, I.; Hydrometallurgy 2002, 63, 85.

[23] Douglas, D. L.; Yost, D. M.; J. Chem. Phys. 1949, 17, 1345.

[24] Donohue, T.; J. Chem. Phys. 1977, 67, 5402.

[25] Qui, L. F.; Kang, X. H.; Wang, T. S.; Separ. Sci. Technol. 1991, 26, 199.

[26] Kusaba, M.; Nakashima, N.; Kawamura, W.; Izawa, Y.; Yamanaka, C.; J. Alloy Compd. 1993, 192, 284.

[27] Hirai, T.; Komasawa, I.; Ind. Eng. Chem. Res. 1995, 34, 237.

[28] Tsushima, S.; Nagasaki, S.; Suzuki, A.; Nucl. Technol. 1997, 118, 42.

[29] de Morais, C. A.; Ciminelli, V. S. T.; Separ. Sci. Technol. 2002, 37, 3305.

[30] Kim, E. H.; Kwon, S. W.; Lee, E. H.; Yoo, J. H.; Korean J. Chem. Eng. 2002, 19, 305.

[31] Donohue, T.; Opt. Eng. 1979, 18, 181. 
[32] Selin, D. L.; Malkov, A. V.; Tarasova, N. P.; Chem. Abstr. 1991, 114, 14693z; USSR Patent SU 1,604,741.

[33] Ferro, S.; De Battisti, A.; J. Electroanal. Chem. 2002, 533, 177.

[34] Zeng, A.; Liu, E.; Tan, S. N.; Zhang, S.; Gao, J.; Electroanal. 2002, 14, 15.

[35] Horowitz, E. P.; Dietz, M. L.; Nelson, D. M.; LaRosa, J. J.; Fairman, W. D.; Anal. Chim. Acta 1990, 238, 263.

[36] Bard, A. J.; Faulkner, L. R.; In Electrochemical Methods: Fundamentals and Applications, 2nd Ed.; John Wiley and Sons, Inc.: New York, 2001, pp 226-260.

[37] Sivaraman, N.; Kumar, R.; Subramaniam, S.; Vasudeve Rao, P. R.; J. Radioanal. Nucl. Chem. 2002, 252, 491.

[38] Sisson, D. H.; Mode, V. A.; Campbell, D. O.; J. Chromatogr. 1972, 66, 129.

[39] Campbell, D. O.; J. Inorg. Nucl. Chem. 1973, 35, 3911.

[40] Schaedel, M.; Trautmann, N.; Herrmann, G.; Radiochim. Acta 1977, 24, 27.

[41] Elchuk, S.; Cassidy, R. M.; Anal. Chem. 1979, 51, 1434.

[42] Hwang, J. M.; Shih, J. S.; Yeh, Y. C.; Wu, S. C.; Analyst 1981, 106, 869.

[43] Mazzucotelli, A.; Dadone, A.; Frache, R.; Baffi, F.; J. Chromatogr. 1985, 349, 137.

[44] Tielrooy, J. A.; Kraak, J. C.; Maessen, F. J. M. J.; Anal. Chim. Acta 1985, 176, 161.

[45] Boissonneau, J. F.; Repellin, M. J.; Eglem, A.; Analysis 1980, 8, 230.

[46] Sevenich, G. J.; Fritz, J. S.; Anal. Chem. 1983, 55, 12. 